\title{
Phylogeny of the supertribe Nebriitae (Coleoptera: Carabidae) based on analyses of molecular sequence data--some surprises.
}

\author{
David H. Kavanaugh ${ }^{\ddagger}$, David R. Maddison, Brian Simisonl, Sean D. Schoville", Joachim Schmidt\#, \\ Arnaud Faille \\ ‡ Californian Academy of Sciences, San Francisco, United States of America \\ $\S$ Oregon State University, Corvallis, United States of America \\ | California Academy of Sciences, San Francisco, United States of America \\ If University of Wisconsin, Madison, WI, United States of America \\ \# University of Rostock, Rostock, Germany \\ a Stuttgart State Museum of Natural History, Stuttgart, Germany
}

Corresponding author: David H. Kavanaugh (dkavanaugh@calacademy.org)

Received: 11 Jun 2019 | Published: 12 Jun 2019

Citation: Kavanaugh D, Maddison D, Simison B, Schoville S, Schmidt J, Faille A (2019) Phylogeny of the supertribe

Nebriitae (Coleoptera: Carabidae) based on analyses of molecular sequence data--some surprises. . ARPHA

Conference Abstracts 2: e37156. https://doi.org/10.3897/aca.2.e37156

\section{Abstract}

To explore phylogenetic relationships among members of the carabid supertribe Nebriitae, we sampled DNA fragments extracted from adults of 244 nebriite species-group taxa, representing about $25 \%$ of known species-group taxa and $93 \%$ (38 of 41 ) of the currently accepted nebriite genus-group taxa. Sequence data were recorded for eight gene fragments (a total of 5654 bases) from nuclear ribosomal, mitochondrial, and nuclear protein coding genes. The resulting phylogeny is based on both individual gene and concatenated gene analyses using Maximum Likelihood and Bayesian methods, which produced nearly identical results. Most nodes are well supported by both high bootstrap and high posterior probability values, and many of them also by unique (autapomorphic) bases.

Many of the previously recognized genus-group taxa are supported as monophyletic, but others are not. Alternative reclassifications that reflect monophyletic groups are still being 
considered, based on the molecular analyses along with considerations of ease of group identification and geographical distribution. Of the two alternative reclassifications being considered, one requires the designation of 16 new subgeneric synonymies and the other only nine new subgeneric synonymies plus the recognition of three new subgenera. However, under both schemes, Nippononebria Uéno (along with its subgenus, Vancouveria Kavanaugh) is re-established as a genus separate from Nebria and sister to Leistus Frölich; and Archastes is embedded within a monophyletic genus Nebria and closely related to subgenera Oreonebria Daniel and Orientonebria Shilenkov. Additional details will be reported in the presentation.

\section{Keywords}

Nebriitae, Ground beetles, DNA, taxonomy, molecular phylogenetics, evolutionary tree

\section{Presenting author}

David H. Kavanaugh

\section{Presented at}

19thECM oral communication 